\title{
Surface Aligned Magnetic Moments and Hysteresis of an Endohedral Single-Molecule Magnet on a Metal
}

\author{
Rasmus Westerström, ${ }^{1,2}$ Anne-Christine Uldry, ${ }^{3}$ Roland Stania, ${ }^{1,3}$ Jan Dreiser, ${ }^{4,3}$ Cinthia Piamonteze, ${ }^{3}$ Matthias Muntwiler, ${ }^{3}$ \\ Fumihiko Matsui, ${ }^{1,5}$ Stefano Rusponi, ${ }^{4}$ Harald Brune, ${ }^{4}$ Shangfeng Yang, ${ }^{6}$ Alexey Popov, ${ }^{7}$ Bernd Büchner, ${ }^{7}$ \\ Bernard Delley, ${ }^{3}$ and Thomas Greber ${ }^{1, *}$ \\ ${ }^{1}$ Physik-Institut, Universität Zürich, Winterthurerstrasse 190, CH-8057 Zürich, Switzerland \\ ${ }^{2}$ Department of Physics and Astronomy, Uppsala University, Box 516, S-751 20 Uppsala, Sweden \\ ${ }^{3}$ Swiss Light Source, Paul Scherrer Institut, CH-5232 Villigen PSI, Switzerland \\ ${ }^{4}$ Institute of Condensed Matter Physics, Ecole Polytechnique Fédérale de Lausanne, CH-1015 Lausanne, Switzerland \\ ${ }^{5}$ Nara Institute of Science and Technology (NAIST), 8916-5 Takayama, Ikoma, Nara 630-0192, Japan \\ ${ }^{6}$ Hefei National Laboratory for Physical Sciences at Microscale, Department of Materials Science and Engineering, \\ University of Science and Technology of China, 96 Jinzhai Road, Hefei 230026, China \\ ${ }^{7}$ Leibniz. Institute of Solid State and Materials Research, Dresden, D-01069 Dresden, Germany
}

(Received 24 June 2014; published 23 February 2015)

\begin{abstract}
The interaction between the endohedral unit in the single-molecule magnet $\mathrm{Dy}_{2} \mathrm{ScN} @ \mathrm{C}_{80}$ and a rhodium (111) substrate leads to alignment of the Dy $4 f$ orbitals. The resulting orientation of the $\mathrm{Dy}_{2} \mathrm{ScN}$ plane parallel to the surface is inferred from comparison of the angular anisotropy of x-ray absorption spectra and multiplet calculations in the corresponding ligand field. The x-ray magnetic circular dichroism is also angle dependent and signals strong magnetocrystalline anisotropy. This directly relates geometric and magnetic structure. Element specific magnetization curves from different coverages exhibit hysteresis at a sample temperature of $\sim 4 \mathrm{~K}$. From the measured hysteresis curves, we estimate the zero field remanence lifetime during $\mathrm{x}$-ray exposure of a submonolayer to be about 30 seconds.
\end{abstract}

DOI: 10.1103/PhysRevLett.114.087201

PACS numbers: 75.50.Xx, 75.70.Ak, 78.70.Dm

A fullerene carbon cage [1] can be used to encapsulate paramagnetic systems consisting of single atoms, as well as small clusters of different composition [2]. A fascinating example is the dysprosium-scandium based endofullereneseries $\mathrm{Dy}_{n} \mathrm{Sc}_{3-n} \mathrm{~N} @ \mathrm{C}_{80}(n=1,2,3)$ where the different stoichiometries result in distinct ground-state properties like tunneling of magnetization $(n=1)$, remanence $(n=2)$, or frustration $(n=3)$ [3-6]. The strong ligand field, mainly due to the central $\mathrm{N}^{3-}$ ion, imposes orientation of the $4 f$ shell and, therefore, noncollinear magnetism. In the case of the di-dysprosium compound $(n=2)$, exchange and dipolar coupling between the two magnetic moments stabilizes hysteresis and a large remanence with a relaxation time of one hour at $2 \mathrm{~K}$ was found [4]. These endofullerenes are, thus, single-molecule magnets (SMMs) [7-11], a class of magnetic compounds with potential for application in spintronics, quantum computing, and high density storage $[12,13]$.

Single-molecule magnets have been studied extensively in the bulk phase for the last two decades, but little is known regarding possible modifications to their intrinsic magnetic properties as the molecules are deposited onto substrates or integrated into different device architectures. This gap in knowledge can largely be attributed to the fragility of most compounds that have restricted research to a few families of molecules. A first proof of principle that molecular nanomagnets can retain their magnetic bistability on a surface was demonstrated for a monolayer (ML) of an $\mathrm{Fe}_{4} \mathrm{SMM}$ on a gold surface [14]. At sub-Kelvin temperatures, the $\mathrm{Fe}_{4}$ SMMs exhibited hysteresis, out-ofplane anisotropy, and quantum tunneling of magnetization (QTM) $[14,15]$. The success of these pioneering experiments is predominantly due to the chemical modification of the $\mathrm{Fe}_{4}$ complex enabling chemically and structurally stable MLs which were prepared ex situ from a solvent under ambient conditions.

Depositing SMMs onto a reactive metal surface, such as ferromagnetic substrates [16,17], requires in situ preparation under ultrahigh vacuum (UHV). In this context, the double-decker complex $\mathrm{TbPc}_{2}$ [18] is the most studied compound. It has been demonstrated that the magnetic anisotropy is preserved at sub-ML coverage on $\mathrm{Cu}(100)$ [19] and that the magnetic moment couples antiferromagnetically to thin nickel films on $\mathrm{Cu}(100)$ and $\mathrm{Ag}(100)$ [16]. Magnetic hysteresis comparable to the bulk phase has been observed in thick molecular films [20]. Only recently, hysteresis with weak remanence was detected in the monolayer regime with graphite [21] and $\mathrm{Si}$ [22] as a substrate, though the influence of the substrate on the magnetic properties is still poorly understood.

Endofullerenes that are synthesized with the KrätschmerHuffmann method are thermally very stable and can be sublimated onto surfaces under UHV. For magnetic endohedral units, the carbon cage acts as a "spin shuttle" that 
protects the spins from chemical interactions. The robustness of the cages also facilitates imaging and manipulation by scanning probes [23-25]. If the cages have a high symmetry, different orientations of the endohedral units are possible. This decreases the average magnetic moment of a system with more than one molecule, and strategies that circumvent this issue are needed for spin alignment. On metallic surfaces, it was shown that endohedral units may order [26]. For the case of $\mathrm{Gd}_{3} \mathrm{~N} @ \mathrm{C}_{80}$ on $\mathrm{Cu}(100)$, a spin system without $4 f$ charge anisotropy, direction dependent magnetic susceptibility was observed, though it could not be directly related to the geometry of the endohedral cluster [27].

Here, we present an x-ray absorption spectroscopy (XAS) study of $\mathrm{Dy}_{2} \mathrm{ScN} @ \mathrm{C}_{80}$ on $\mathrm{Rh}(111)$. In the first layer, the endohedral units and their magnetic moments align with the metal substrate, which is inferred from the angle dependence in XAS and x-ray magnetic circular dichroism (XMCD). Hysteresis curves demonstrate that the proximity of the metal surface has a pronounced influence on the magnetic bistability. Compared to thicker films, which are representative for the bulk phase, the smaller opening of the hysteresis for the sub-ML indicates faster magnetic relaxation times.

The molecules [see Fig. 1(a)] have been sublimated in situ onto the clean $\mathrm{Rh}(111)$ substrate, following the recipe in Ref. [26]. The sample was cooled in zero magnetic field and the layer thickness is estimated from the X-ray absorption of Dy [28]. The XAS measurements were performed at the X-Treme beam line [32] of the Swiss Light Source. Absorption spectra were acquired by recording the total electron yield in the on-the-fly mode [33], at sample temperatures of $\sim 4 \mathrm{~K}$ and with an external magnetic field applied along the $\mathrm{x}$-ray beam.

Figure 2(a) shows $\mathrm{x}$-ray absorption for a sub-ML coverage of $\mathrm{Dy}_{2} \mathrm{ScN} @ \mathrm{C}_{80}$ on $\mathrm{Rh}(111)$ as a function of the angle $\theta$ between surface normal and x-ray beam [see Fig. 1(b)]. The data were recorded over the Dy $M_{5}$ edge $\left(3 d_{5 / 2} \rightarrow 4 f\right)$ using right $\left(I^{+}\right)$and left $\left(I^{-}\right)$circular polarized $\mathrm{x}$ rays. After background subtraction, the XAS $\left(I^{+}+I^{-}\right)$were normalized to the integrated $M_{5}$ absorption signal in order to compensate for the angular dependence
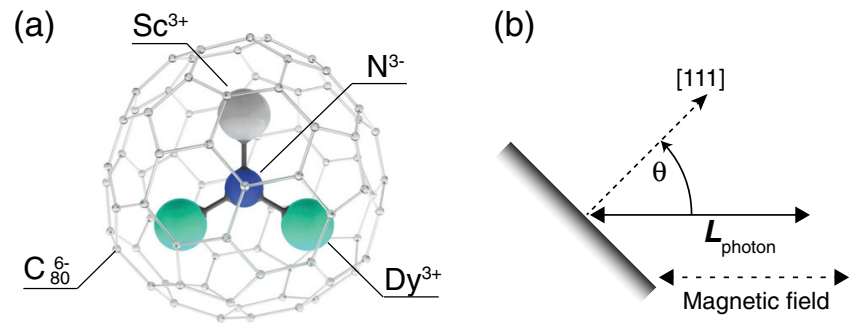

FIG. 1 (color online). (a) Ball-and-stick model of $\mathrm{Dy}_{2} \mathrm{ScN} @ \mathrm{C}_{80}$. (b) Measurement geometry with the angular momentum of the x rays $L_{p h}$, parallel or antiparallel to the magnetic field and at an angle of $\theta$ with respect to the normal of the $\mathrm{Rh}(111)$ surface. of the total electron yield. A significant change in shape of the XAS multiplet spectra is observed as the sample is rotated from normal $\theta=0^{\circ}$ to a larger angle of incidence $\theta=70^{\circ}$. This anisotropy in the XAS is attributed to an anisotropic distribution of the $4 f$ electron charges due to their interaction [34] with the ligand field of the central nitrogen ion, the neighbor rare earth ions, and the $\mathrm{C}_{80}$ cage. This effect would not be present for an isotropic distribution of the endohedral $\mathrm{Dy}_{2} \mathrm{ScN}$ units and, therefore, indicates a preferred orientation of the Dy $4 f$ orbitals with respect to the surface.

The orientation of the Dy $4 f$ orbitals may be inferred from comparison of the experiments with multiplet calculations. The crystal- or ligand-field multiplet theory for the circularly polarized $\mathrm{x}$-ray absorption is a continuation in a long history of conceptually fairly similar [35], close to first principles, calculations with semiempirical parameters to fine tune the fit to experiment. This circumvents unfeasible calculations comprising the coupling to a huge number of electron states of lesser importance for the appearance of the spectrum. The endohedral Dy ions are trivalent $\left(\mathrm{Dy}^{3+}\right)$, which leads to a $4 f^{9}$ ground-state configuration. The final state in the present absorption spectra is, consequently, $3 d^{9} 4 f^{10}$. The ligand field determines the easy axis with a twofold degenerate ground state $\pm J_{z}$. The magnetic field lifts this degeneracy, and induces dichroism [36]. For low magnetic fields, we find a very small influence on the XAS.
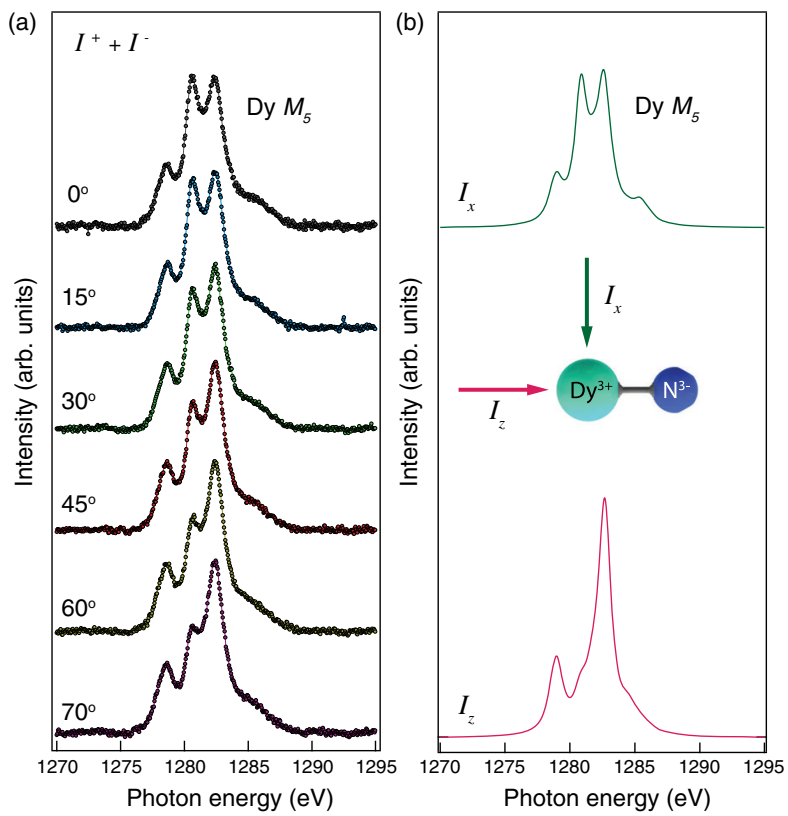

FIG. 2 (color online). (a) XAS measured at the Dy $M_{5}$ edge from a sub-ML of $\mathrm{Dy}_{2} \mathrm{ScN} @ \mathrm{C}_{80} / \mathrm{Rh}(111) T=4 \mathrm{~K}$, $\mu_{0} H=6.5 \mathrm{~T}$, measurement geometry in Fig. 1(b). Each data set is normalized to the integrated intensity. (b) Calculated absorption with the x-ray beam and external field oriented parallel $I_{z}$, and perpendicular $I_{x}$, to the magnetic easy axis (Dy-N bond). 
The ligand field, here from a point charge model of the $\left[\mathrm{Dy}_{2}^{3+} \mathrm{Sc}^{3+} \mathrm{N}^{3-}\right]^{6+}$ ion, describes the site symmetry [28]. Figure 2(b) displays calculated XAS spectra from the Dy $M_{5}$ edge with the x-ray beam and an external magnetic field of 6.5 T applied parallel $\left(I_{z}\right)$ and perpendicular $\left(I_{x}\right)$ to the Dy-N bond. The resemblance of the calculated $I_{x}$ spectrum and data measured at normal incidence $\left(\theta=0^{\circ}\right)$, thus, indicates that the endohedral units adopt an orientation parallel to the surface.

The magnetism of the system is governed by the spin and orbital moment of the Dy $4 f$ electrons that have a total magnetic moment of $10 \mu_{B}$ per $\mathrm{Dy}^{3+}$ ion $[3,4]$. Any anisotropy in the spin and orbital moments will give rise to a polarization dependent absorption at the Dy $M_{5}$ edge and an XMCD spectrum $\left(I^{+}-I^{-}\right)_{M_{5}}$. The magnitude of the $\mathrm{XMCD}$ signal is given by the projection of the corresponding magnetic moment $\vec{\mu}_{i}$ of the absorbing dysprosium ion $i$ onto the direction of the impinging $\mathrm{x}$ rays $\vec{k}$ [37]

$$
I_{\mathrm{XMCD}} \propto \vec{\mu}_{i} \cdot \vec{k}
$$

For an isotropic system, where the magnetic moments are either randomly distributed or aligned to the external magnetic field, the resulting XMCD signal in the present measurement geometry is independent of the incidence angle. Any macroscopic magnetic anisotropy is reflected in different XMCD spectra as a function of incidence angle. Polarization dependent XAS and corresponding XMCD spectra are shown in Figs. 3(a) and 3(b) for incidence angles $\theta$ of $0^{\circ}$ and $60^{\circ}$, respectively. Comparison of the two spectra reveals a significant angle dependence which indicates a macroscopic magnetic anisotropy in the sub-ML. Electrostatic interaction with the surrounding ligands, in particular the central $\mathrm{N}^{3-}$ ion, results in a strong axial anisotropy which restricts the individual Dy moments $\vec{\mu}_{i}$ to orient parallel, or antiparallel, to the corresponding magnetic easy axis directed along the $\mathrm{Dy}_{i}-\mathrm{N}$ bonds $[3-6,38]$. The observed magnetic ordering is, thus, directly related to the axial anisotropy of the individual Dy ions and the preferred adsorption geometry of the endohedral cluster, which must be imposed by the surface.

The magnetic anisotropy is quantified in Fig. 3(c), where the XMCD angular dependence is shown. This confirms that the dysprosium moments are predominantly oriented parallel to the surface. A small out-of-plane fraction is inferred from the nonvanishing dichroism at $\theta=0^{\circ}$. The observed behavior can be modeled by assuming a Gaussian distribution of the magnetic moments centered in the surface plane $\left(\theta=90^{\circ}\right)$. The fit yields a distribution of $90^{\circ} \pm 16^{\circ}$. This implies that the Dy-N bonds are not completely parallel to the surface, which is in line with a resonant $\mathrm{x}$-ray photoelectron diffraction study performed on a ML of $\mathrm{Dy}_{3} \mathrm{~N} @ \mathrm{C}_{80}$ on $\mathrm{Cu}(111)$, where the room temperature data indicated a coexistence of planar (a)

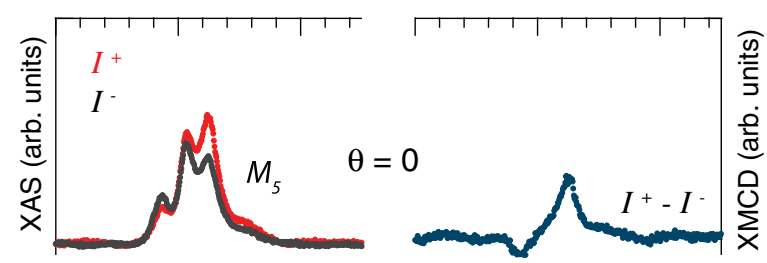

(b)
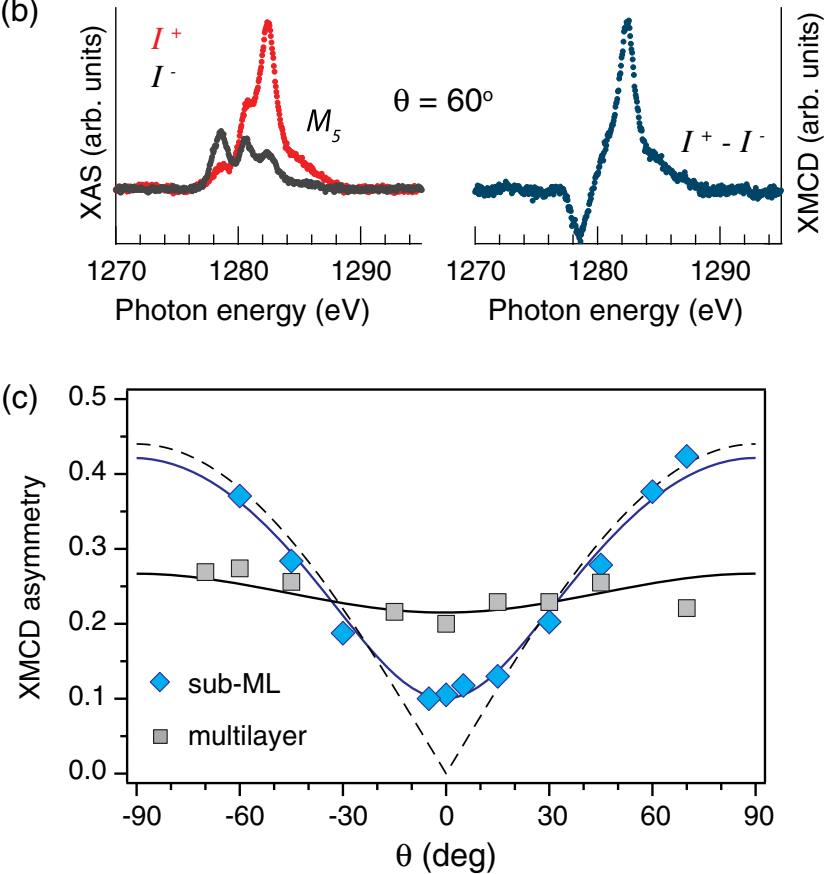

FIG. 3 (color online). Sub-ML of $\mathrm{Dy}_{2} \mathrm{ScN} @ \mathrm{C}_{80} / \mathrm{Rh}(111)$, $T=4 \mathrm{~K}, \mu_{0} H=6.5 \mathrm{~T}$, measurement geometry of Fig. 1(b). The polarization dependent XAS spectra (left panel), and the corresponding XMCD spectra (right panel), were measured at an incidence angle of $\theta=0^{\circ}$ (a) and $\theta=60^{\circ}$ (b). (c) Angle dependence of the integrated XMCD signal normalized to the integrated XAS over the Dy $M_{5}$ edge. The dashed line corresponds to the expected angle dependence for magnetic moments oriented parallel to the surface, whereas the blue line takes into account a Gaussian distribution, centered in the surface plane and with a standard deviation of $16^{\circ}$.

endohedral units inclined to the surface, and slightly pyramidal configurations parallel to the surface [26].

The square symbols in Fig. 3(c) correspond to the same measurements performed on a multilayer containing 7 times more molecules [28]. The weak angular anisotropy observed is attributed to the residual influence of the surface.

The relaxation time of the magnetic moments in a given environment is a key property of single-molecule magnets. Relaxation times that are slow compared to the time scale of the measurement will result in magnetic hysteresis. Figure 4 shows element specific magnetization curves from the Dy $M_{5}$ edge at a field sweep rate of $2 \mathrm{~T} / \mathrm{min}$ with the $\mathrm{x}$ rays and the magnetic field at an angle of $60^{\circ}$ with respect to the surface normal. A significant hysteresis is observed for both systems [28] demonstrating that the corresponding relaxation times are slow compared to the measurement 

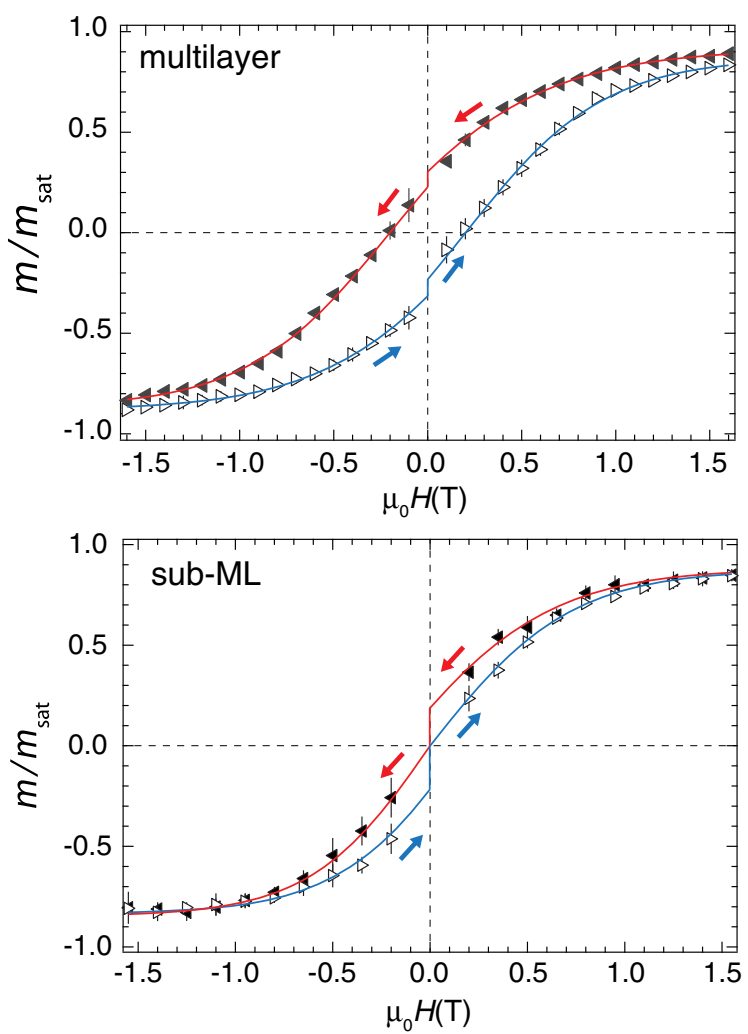

FIG. 4 (color online). Hysteresis curves measured from a multilayer and a sub-ML of $\mathrm{Dy}_{2} \mathrm{ScN} @ \mathrm{C}_{80} / \mathrm{Rh}(111)$ at a magnetic field sweep rate of $2 \mathrm{~T} / \mathrm{min}$ and a sample temperature of $\sim 4 \mathrm{~K}$. The $\mathrm{x}$-ray flux was $5 \times 10^{10}$ photons $/ \mathrm{mm}^{2} / \mathrm{s}$. The data were recorded with the $\mathrm{x}$-ray beam and the magnetic field at an incidence angle of $\theta=60^{\circ}$. The magnetization curves correspond to the average of several independent measurements, where the error bars are the standard deviation at each external magnetic field. The arrows indicate the ramping direction of the magnetic field, the lines are guides to the eye, and $m_{\text {sat }}$ is the saturated value at $\pm 6.5 \mathrm{~T}$. The drop in magnetization at zero field is a consequence of the time of $30 \mathrm{~s}$ it takes for the magnet to switch polarity.

time. However, comparing the magnetization curves from the two systems indicates that the magnetic bistability of $\mathrm{Dy}_{2} \mathrm{ScN} @ \mathrm{C}_{80}$ is modified by the proximity of the rhodium metal surface.

Single-ion $4 f$ magnets, such as, e.g., $\mathrm{TbPc}_{2}$, exhibit poor remanence due to the rapid decay of the magnetization at low fields through QTM. In contrast, for bulk samples of $\mathrm{Dy}_{2} \mathrm{ScN} @ \mathrm{C}_{80}$, an exchange and dipole barrier of $0.96 \pm$ $0.1 \mathrm{meV}$ suppresses QTM that in turn leads to a significant remanence and coercive field [4]. This is clearly observed in the multilayer system, where the drop in magnetization at zero field is attributed to the delay of $30 \mathrm{~s}$ when changing the polarity of the magnet. From the $25 \%$ decrease in magnetization during these $30 \mathrm{~s}$, we derive a remanence relaxation time of $110 \mathrm{~s}$ in the multilayer system. Compared to bulk samples in the dark [4], this is about 4 times faster, and mainly related to $\mathrm{x}$-ray induced demagnetization [39].
The remanence time of the submonolayer system is still shorter because the magnetization vanishes during the switching of the magnet. From the comparison of the two magnetization curves we can estimate the remanence time for the sub-ML. Here, we assume that the ratio of the hysteresis openings (Fig. S3 [28]), recorded for a fixed temperature and field sweep rate, is a measure of the relaxation times in the two systems. Under this assumption, we obtain a 4 times faster relaxation rate in the sub-ML and a remanence time under $\mathrm{x}$-ray irradiation at $4 \mathrm{~K}$ of about $30 \mathrm{~s}$.

The shorter remanence time of the sub-ML may be related to residual interaction of the Rh Fermi sea across the $\mathrm{C}_{80}$ shell. Furthermore, since the Dy magnetic dipoles lie in a plane, their interaction is stronger, which may also accelerate demagnetization. Also, at sub-ML coverage, the total electron yield below the $M_{5}$ edge is about $10 \%$ higher than in the multilayer case and the reduced bistability could, therefore, be demagnetization due to secondary electrons from the substrate [39]. However, the opening of the hysteresis demonstrates that the rate at which the magnetization relaxes to its equilibrium value is still slow compared to the measurement time.

In summary, angle dependent XAS from a sub-ML of $\mathrm{Dy}_{2} \mathrm{ScN} @ \mathrm{C}_{80}$ on $\mathrm{Rh}(111)$ reveals a one-to-one correspondence between structural and magnetic ordering: The combined effect of the local magnetic easy axis for the encapsulated Dy ions, and the preferred absorption geometry of the endohedral cluster, indicates surface aligned $4 f$ moments and a macroscopic anisotropy. At a sample temperature of $\sim 4 \mathrm{~K}$ we observe a hysteresis in the subML. Although orientational ordering of endohedral molecules at surfaces, as well as magnetic hysteresis of bulk samples of such molecules have been shown, we demonstrate here the structural, and magnetic ordering of surface adsorbed endohedral molecules creating a stable macrospin for molecular submonolayers. This is observed at a 1 order of magnitude higher sample temperature than previously reported for the $3 d$-based $\mathrm{Fe}_{4}$ SMMs [14,15] and, thus, paves the way for many more experiments, like scanning tunneling spectroscopy on single molecules.

We gratefully acknowledge financial support from the Swiss National Science Foundation (SNF Projects No. 200021L-147201, No. 200021-129861, No. PZ00P2-142474), the Swedish Research Council (Grant No. 350-2012-295) and the Deutsche Forschungsgemeinschaft (DFG Project No. PO 1602/1-1).

*greber@physik.uzh.ch

[1] H. W. Kroto, J. R. Heath, S. C. O’ Brien, R. F. Curl, and R. E. Smalley, Nature (London) 318, 162 (1985).

[2] A. Popov, S. Yang, and L. Dunsch, Chem. Rev. 113, 5989 (2013).

[3] R. Westerström et al., J. Am. Chem. Soc. 134, 9840 (2012).

[4] R. Westerström et al., Phys. Rev. B 89, 060406(R) (2014). 
[5] V. Vieru, L. Ungur, and L. F. Chibotaru, J. Phys. Chem. Lett. 4, 3565 (2013).

[6] F. Cimpoesu, Nita Dragoe, H. Ramanantoanina, W. Urland, and C Daul, Phys. Chem. Chem. Phys. 16, 11337 (2014).

[7] R. Sessoli, D. Gatteschi, A. Caneschi, and M. A. Novak, Nature (London) 365, 141 (1993).

[8] D. Gatteschi et al., Molecular Nanomagnets (Oxford University Press, New York, 2006).

[9] F. Habib, and M. Murugesu, Chem. Soc. Rev. 42, 3278 (2013).

[10] D. N. Woodruff, R. E. P. Winpenny, and R. A. Layfield, Chem. Rev. 113, 5110 (2013).

[11] P. Zhang, Y.-N. Guo, and J. Tang, Coord. Chem. Rev. 257, 1728 (2013).

[12] M. N. Leuenberger, and D. Loss, Nature (London) 410, 789 (2001).

[13] L. Bogani, and W. Wernsdorfer, Nat. Mater. 7, 179 (2008).

[14] M. Mannini et al., Nat. Mater. 8, 194 (2009).

[15] M. Mannini et al., Nature (London) 468, 417 (2010).

[16] A. Lodi Rizzini et al., Phys. Rev. Lett. 107, 177205 (2011).

[17] J. Dreiser et al., ACS Nano, 8, 4662 (2014).

[18] N. Ishikawa, M. Sugita, T. Ishikawa, S.-y. Koshihara, and Y. Kaizu, J. Am. Chem. Soc. 125, 8694 (2003).

[19] S. Stepanow et al., J. Am. Chem. Soc. 132, 11900 (2010).

[20] L. Margheriti et al., Adv. Mater. 22, 5488 (2010).

[21] D. Klar, A. Candini, L. Joly, S. Klyatskaya, B. Krumme, P. Ohresser, J.-P. Kappler, M. Ruben, and H. Wende, Dalton Trans. 43, 10686 (2014).

[22] M. Mannini et al., Nat. Commun. 5, 4582 (2014).

[23] M. J. Butcher, J. W. Nolan, M. R. C. Hunt, P. H. Beton, L. Dunsch, P. Kuran, P. Georgi, and T. J. S. Dennis, Phys. Rev. B 67, 125413 (2003).

[24] T. Huang, J. Zhao, M. Feng, A. A. Popov, S. Yang, L. Dunsch, and H. Petek, Chem. Phys. Lett. 552, 1 (2012).

[25] Y. Yasutake, Z. Shi, T. Okazaki, H. Shinohara, and Y. Majima, Nano Lett. 5, 1057 (2005).
[26] M. Treier, P. Ruffieux, R. Fasel, F. Nolting, S. Yang, L. Dunsch, and T. Greber, Phys. Rev. B 80, 081403(R) (2009).

[27] C. F. Hermanns et al., Phys. Rev. Lett. 111, 167203 (2013).

[28] See Supplemental Material at http://link.aps.org/ supplemental/10.1103/PhysRevLett.114.087201 for details regarding the multilplet calculations, coverage determination, and further analysis of the magnetization curves that includes Refs. [29-31].

[29] S. Berner et al., Angew. Chem., Int. Ed. Engl. 46, 5115 (2007).

[30] F. C. Vicentin, S. Turchini, F. Yubero, J. Vogel, and M. Sacchi, J. Electron Spectrosc. Relat. Phenom. 74, 187 (1995).

[31] M. M. Sant Anna, A. S. Schlachter, G. Öhrwall, W. C. Stolte, D. W. Lindle, and B. M. McLaughlin, Phys. Rev. Lett. 107, 033001 (2011).

[32] C. Piamonteze et al., J. Synchrotron Radiat. 19, 661 (2012).

[33] J. Krempaský, U. Flechsig, T. Korhonen, D. Zimoch, Ch. Quitmann , F. Nolting, AIP Conf. Proc. 1234, 705 (2010).

[34] F. Groot and A. Kotani, Core Level Spectroscopy of Solids (OCR Press, 2008).

[35] Contrary to earlier approaches, our method treats S-O, electron-electron, and crystal field at the same level in a single variation.

[36] A. Uldry, F. Vernay, and B. Delley, Phys. Rev. B, 85, 125133 (2012).

[37] J. Stöhr and H. C. Siegmann, Magnetism: From Fundamentals to Nanoscale Dynamics (Springer-Verlag Berlin, 2006).

[38] M. Wolf, K.-H. Müller, D. Eckert, Y. Skourski, P. Georgi, R. Marczak, M. Krause, and L. Dunsch, J. Magn. Magn. Mater. 290-291, 290 (2005).

[39] J. Dreiser, R. Westerström, C. Piamonteze, F. Nolting, S. Rusponi, H. Brune, S. Yang, A. Popov, L. Dunsch, and T. Greber, Appl. Phys. Lett. 105, 032411 (2014). 


\section{Supporting information for the manuscript "Surface aligned magnetic moments and hysteresis of an endo- hedral single-molecule magnet on a metal"}

Rasmus Westerstöm et al.

\section{Multiplet calculations}

The ligand electrostatic field model is fully defined by the parameters in Table S1 (The small contributions from the $\mathrm{C}_{80}^{6-}$ were neglected). It has a decisive influence on the orientation of the magnetic moment. Its strength has very little impact on the shape and intensity of the spectrum in this case. The point charges are an approximation to the actual electrostatics. Moreover, this field also models the symmetry breaking due to the hybridization of the orbitals, which is only remotely related to the electrostatics. Because of this insensitivity, the ligand (crystal) field is left with unity scaling. For a good semiempirical fit we scale electron-electron interactions by 0.76 , spin-orbit coupling by 0.96 , use a core hole broadening of $0.53 \mathrm{eV}$ and apply a corrective shift of $-0.6 \mathrm{eV}$ to the XAS spectrum. The B field is taken in the weak limit.

Table S1: Coordinates (x, y, z) and charges (q) of the ligand field used for the calculation of the Dy $3 d^{9} 4 f^{10}$ multiplets. The Dy ion sits at the origin $(\mathrm{x}=0, \mathrm{y}=0, \mathrm{z}=0)$.

\begin{tabular}{|c|c|c|c|c|}
\hline $\mathrm{x}$ & $\mathrm{y}$ & $\mathrm{z}$ & $\mathrm{q}$ & element \\
\hline 0.00 & 0.0 & 2.03 & -3 & $\mathrm{~N}$ \\
\hline 0.00 & 1.74 & 3.09 & +3 & $\mathrm{RE}$ \\
\hline 0.00 & -1.74 & 3.09 & +3 & $\mathrm{RE}$ \\
\hline
\end{tabular}

\section{X-ray absorption (XAS)}

Figure S1 shows the normalized total electron yield (TEY) at the Dy $M_{4,5}$-edge from the sub-ML and the multilayer $\left(T \sim 4 \mathrm{~K}, \mu_{0} H=6.5 \mathrm{~T}\right)$. The integrated intensity of the multilayer is about seven times larger than that of the sub-ML.

\section{Quantification of the coverage}

The $\mathrm{Dy}_{2} \mathrm{ScN} @ \mathrm{C}_{80}$ coverage was quantified from comparison of Dy $M_{5}$ and $\mathrm{N} K$ XAS of a single layer of boron nitride on $\operatorname{Rh}(111)$, where the nitrogen atom density is precisely known [1]. To this end we used the XAS normalized with the gold mesh current, the Au XAS cross sections at the given photon energies and the experimental resonant absorption cross sections of Dy $M_{5}[2]$ and $\mathrm{N} K$ [3]. The $\mathrm{N} K$ cross section was reduced according to a partial filling of the $\mathrm{N} 2 \mathrm{p}$ shell in $h$-BN of 3.5 instead of 3 p-electrons for the nitrogen atom. With this we get a coverage of about $3 \mathrm{ML}$ for the multilayer and about 0.5 monolayer for the submonolayer. 

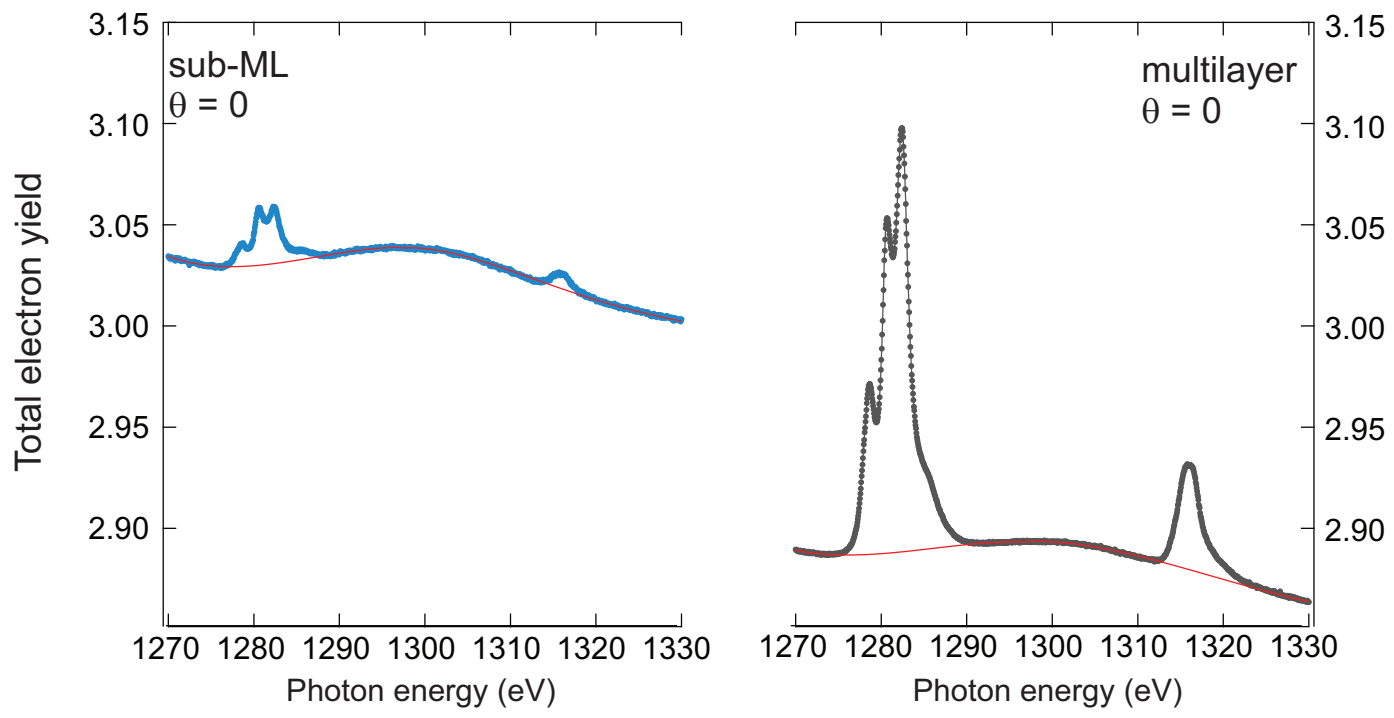

Figure S1: XAS $\left(I^{+}+I^{-}\right)$recorded at normal incidence $\left(\theta=0^{\circ}\right)$ at $\sim 4 \mathrm{~K}$ and $6.5 \mathrm{~T}$ over the Dy $\mathrm{M}_{4,5}$ edge from the sub-ML and the multilayer preparation. The total electron yield is normalized with the gold mesh current.

\section{XMCD of the thick layers}

Figure S2 displays polarization dependent XAS and corresponding XMCD at normal (a) and gracing (b) incidence angle for the multilayer $\left(T \sim 4 \mathrm{~K}, \mu_{0} H=6.5 \mathrm{~T}\right)$.

\section{Hystereses loops}

The significance of the hysteresis loops is shown in Figure S3 with the difference in magnetisation between the two branches of the hysteresis curves in Figure 4 of the main manuscript. Clearly, both systems display hysteresis.

\section{References}

[1] S. Berner et al, Angew. Chem. Int. Ed. 47, 5115 (2007)

[2] F.C. Vicentin et al, Journal of Electron Spectroscopy and Related Phenomena 74, $187(1995)$

[3] M. M. Sant Anna et al, PRL 107, 033001 (2011) 

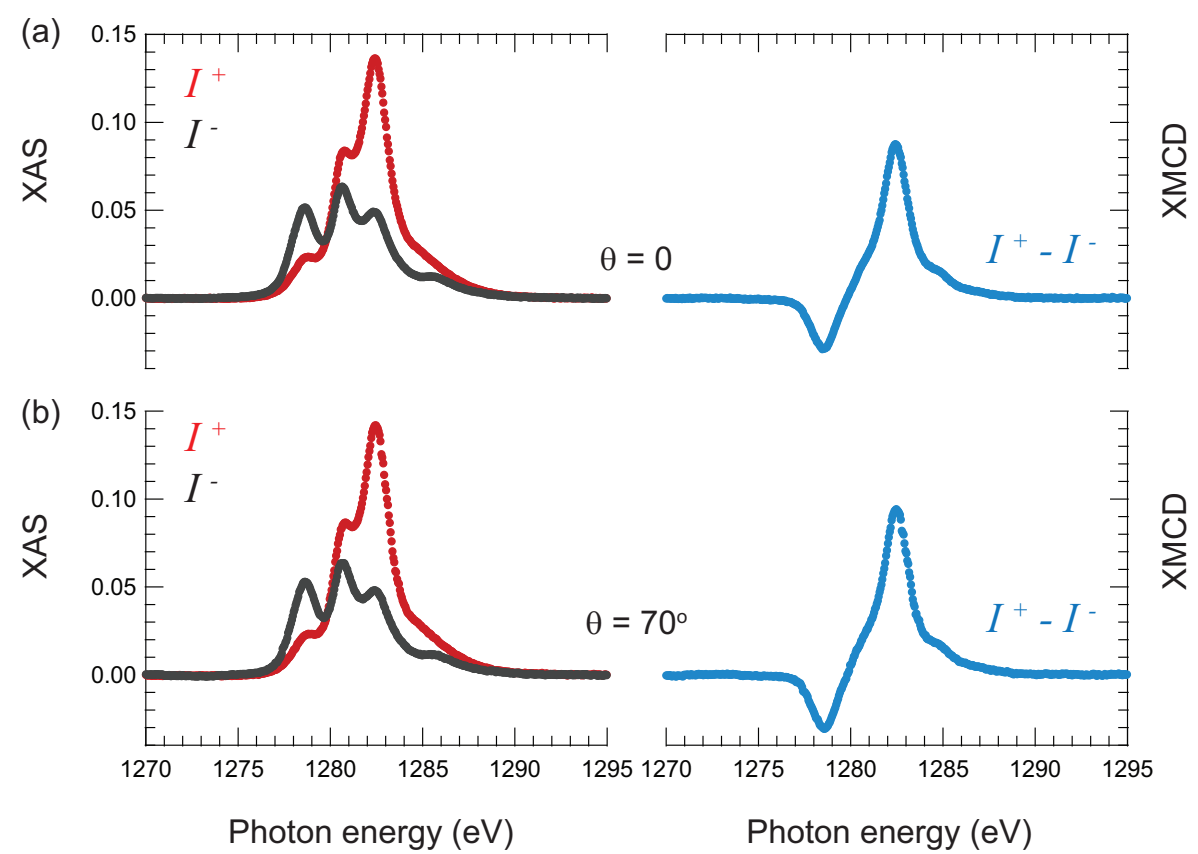

Figure S2: X-ray absorption from a multilayer of $\mathrm{Dy}_{2} \mathrm{ScN}_{\mathrm{C}} \mathrm{C}_{80}$ on $\mathrm{Rh}(111), T \sim 4 \mathrm{~K}$, $\mu_{0} H=6.5 \mathrm{~T}$, measurement geometry of Fig. 1 (b). The polarization dependent XAS spectra (left panel), and the corresponding XMCD spectra (right panel), were measured at an incidence angle of $\theta=0^{\circ}$ (a) and $\theta=70^{\circ}$ (b). Each data set is normalized to the integrated absorption at the Dy $\mathrm{M}_{5}$ edge. 


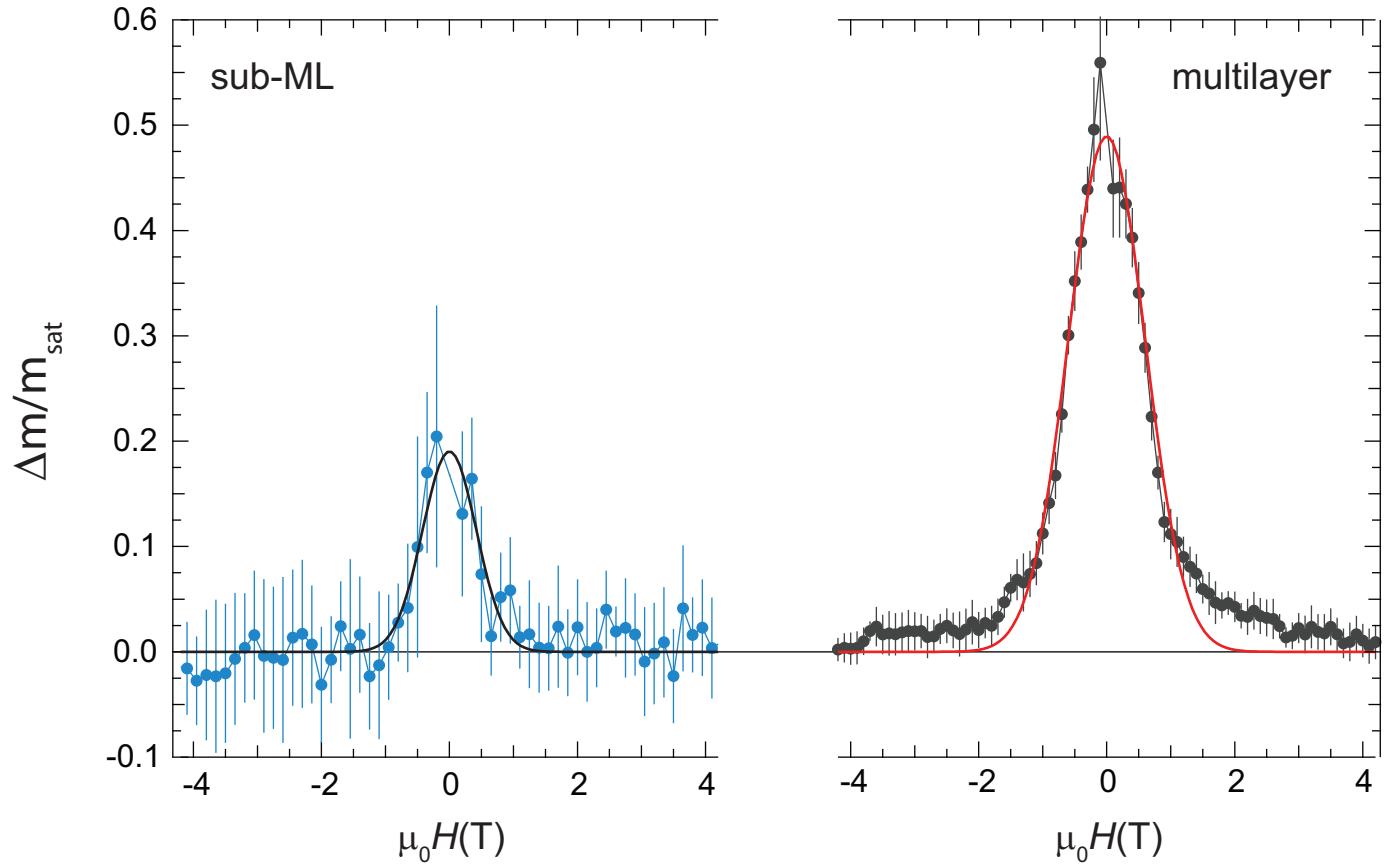

Figure S3: The difference in XMCD at each external magnetic field value for the two field sweep directions in the magnetization curves in Fig. 4 of the main manuscript. The data are normalized to the saturation magnetization at $\pm 6.5 \mathrm{~T}$. The integrated area for the sub-ML and the multilayer is $0.2 \mathrm{~T} \cdot \mathrm{m}_{\text {sat }}$ and $0.74 \mathrm{~T} \cdot \mathrm{m}_{\text {sat }}$, respectively. The FWHM is $0.99 \pm 0.08 \mathrm{~T}$ for the sub-ML and $1.41 \pm 0.03 \mathrm{~T}$ for the multilayer. 